\title{
Gate-controlled BCS-BEC crossover in a two-dimensional superconductor
}

\author{
Yuji Nakagawa ${ }^{1,2}$, Yuichi Kasahara ${ }^{3}$, Takuya Nomoto ${ }^{1}$, Ryotaro Arita $^{1,4}$, Tsutomu Nojima ${ }^{5}$, \\ Yoshihiro Iwasa ${ }^{1,2,4 *}$
}

${ }^{1}$ Department of Applied Physics, University of Tokyo, Hongo 7-3-1, Bunkyo-ku, Tokyo 113-8656, Japan. ${ }^{2}$ Quantum-Phase Electronics Center, University of Tokyo, Hongo 7-3-1, Bunkyo-ku, Tokyo 113-8656, Japan. ${ }^{3}$ Department of Physics, Kyoto University, Kitashirakawa Oiwakecho, Sakyo-ku, Kyoto 606-8502, Japan. ${ }^{4}$ RIKEN Center for Emergent Matter Science, Hirosawa 2-1, Wako, Saitama 351-0198, Japan. Institute for Materials Research, Tohoku University, Katahira 2-1-1, Aoba-ku, Sendai 980-0812, Japan.

*Corresponding author. Email: iwasa@ap.t.u-tokyo.ac.jp

\begin{abstract}
Bardeen-Cooper-Schrieffer (BCS) superfluidity and Bose-Einstein condensation (BEC) are the two extreme limits of the ground state of the paired fermion systems. We report crossover behavior from the BCS limit to the BEC limit realized by varying carrier density in a two-dimensional (2D) superconductor, electrondoped layered material ZrNCl. The phase diagram, established by simultaneous measurements of resistivity and tunneling spectra under ionic gating, demonstrates a pseudogap phase in the low doping regime. The ratio of the superconducting transition temperature and Fermi temperature in the low carrier density limit is consistent with the theoretical upper bound expected in the BCS-BEC crossover regime. These results indicate that the gate-doped semiconductor provides an ideal platform for the 2D BCS-BEC crossover without added complexities present in other solid-state systems.
\end{abstract}

The pairing and condensation of fermions are fundamental to a variety of systems, ranging from neutron stars to superconductors and ultracold atomic gases $(1-7)$. There are two limiting cases for fermion condensation, described by two distinct theories: the Bardeen-Cooper-Schrieffer (BCS) theory and Bose-Einstein condensation (BEC). The former explains superfluidity in the weak-coupling or high-density limit, where individual fermions directly condense to a coherent state of fermion pairs. This type of condensation is typically observed in the superconductivity (SC) of electrons. The latter occurs in the strong-coupling, low-density limit. Fermions first form pairs that behave as bosons, and these bosons then undergo the BEC to the superfluid state (1). This phenomenon is most clearly seen in fermionic gases (3). These two limits are continuously connected through an intermediate regime, exhibiting universal behavior called the BCS-BEC crossover (2, 7).

Ultracold atomic gases and superconductors are experimental settings favorable for the observation of the BCSBEC crossover because the coupling strength between the constituent fermions can be controlled in a quasicontinuous manner. In ultracold atomic gasses, the coupling strength is highly modulated via Feshbach resonances (7), sweeping through the crossover regime from the BEC limit $(4,6)$. In superconductors, the crossover regime can be en- tered from the BCS limit by controlling the carrier density and, consequently, the coupling strength.

The dimensionless coupling strength in superconductors is determined by $\Delta / E_{\mathrm{F}}$, where $\Delta$ is the superconducting gap, and $E_{\mathrm{F}}$ is the Fermi energy measured from the bottom of the conduction band. As $\Delta / E_{\mathrm{F}}$ is increased by enhanced pairing interaction or reduced carrier density, the system enters the BCS-BEC crossover regime accompanied by the enhancement of $T_{\mathrm{c}} / T_{\mathrm{F}}$, where $T_{\mathrm{c}}$ and $T_{\mathrm{F}}$ are the superconducting critical temperature and the Fermi temperature, respectively. As represented in the well-known Uemura plot (8), conventional metallic superconductors, such as $\mathrm{Nb}$ and $\mathrm{Al}$, reside deep inside the BCS limit $\left(T_{\mathrm{c}} / T_{\mathrm{F}}<<1\right)$, whereas the "exotic" superconductors, including cuprates, organics, heavy fermions, and iron-based superconductors, are located rather close to the BCS-BEC crossover region with $T_{\mathrm{c}} / T_{\mathrm{F}} \sim$ 0.05. However, even in these systems, the coupling strengths are not high enough to reach the BEC limit beyond the crossover regime; additionally, in the low carrier density, strong electron correlation effects and magnetic ordering (9) cloud the crossover phenomena by added complexity. Therefore, the clear demonstration of the BCS-BEC crossover is still a key challenge in the study of SC. Although FeSe systems (10-12), magic-angle twisted bilayer graphene (13, 14), and layered nitrides (15) have been studied in this context, 
the wide-range control of $\Delta / E_{\mathrm{F}}$ needed to go from the BCS regime to the BEC limit has not been realized to date.

Here we study the superconductor $\mathrm{Li}_{x} \mathrm{ZrNCl}$, a lithiumintercalated layered nitride $(15,16)$ (Fig. 1A). Lithium supplies electrons to the double honeycomb $\mathrm{ZrN}$ layer, which is a band insulator in the absence of doping. A single conduction band originating from $\mathrm{Zr} 4 d$ orbitals hybridized with $\mathrm{N}$ $2 p$ orbitals exists at each corner of the hexagonal Brillouin zone ( $\mathrm{K}$ and $\mathrm{K}^{\prime}$ points). A unique property reported in polycrystalline bulk samples of $\mathrm{Li}_{x} \mathrm{ZrNCl}$ is the $T_{\mathrm{c}}$ enhancement with decreased doping until SC is quenched by disorder (17). Further increase of $T_{\mathrm{c}}$ above $15 \mathrm{~K}$ is theoretically proposed to exist in the low-carrier-density limit $(18,19)$, which corresponds to a substantial increase of $\Delta / E_{\mathrm{F}}$ and the realization of the BCS-BEC crossover.

Single-crystal measurements of pristine $\mathrm{ZrNCl}$ have been performed by using ionic gating methods $(20,21)$. Interface SC was realized in electrostatic electric double layer transistors (EDLTs), where the intrinsic two-dimensional (2D) nature of SC has been demonstrated in this 2D material. Recently, we introduced a modified device structure designed for an electrochemical intercalation mode as well as the tunneling spectroscopy measurements (15). The electrical control of the Li amount in single crystals has been proven to be more effective in pursuing the low carrier density limit of $\mathrm{Li}_{x} \mathrm{ZrNCl}$, as well as of $\mathrm{Li}_{x} \mathrm{HfNCl}$, than the conventional chemical intercalation methods in polycrystalline samples using $n$-BuLi. A dimensional crossover from anisotropic three-dimensional (3D) to 2D SC was found to occur with decreasing the carrier density (15).

Here, we report the superconducting behavior of $\mathrm{Li}_{x} \mathrm{ZrNCl}$ in an even lower carrier density regime down to $x$ $=0.0038$. With diluting the carrier density, $T_{\mathrm{c}}$ shows the maximum value of $19.0 \mathrm{~K}$ at $x=0.011 . \Delta / E_{\mathrm{F}}$ increases above 0.3 , which is considered to be the border between the weakcoupling BCS limit and the BCS-BEC crossover regime in 2D systems (22). The Berezinskii-Kosterlitz-Thouless (BKT) transition temperature $T_{\mathrm{BKT}}$ reaches $0.12 T_{\mathrm{F}}$, which is close to the maximum value generally predicted in the fermion systems, especially in the BCS-BEC crossover regime (23). Tunneling spectroscopy simultaneously performed with the resistivity measurements reveals that the onset temperature of the pseudogap phase at which the fermions form pairs without condensation $(6,7)$, is higher in the diluted regime. These results are an unambiguous demonstration of a crossover from the BCS to BEC regime of a $2 \mathrm{D}$ superconductor by scanning the doping level across nearly two orders of magnitude from $x=0.28$ to 0.0038 .

Figure 1B presents a schematic of our ionic-gating device structure. In addition to the Hall bar structure, narrow electrodes for the tunneling spectroscopy were prepared on the channel region between the source and drain electrodes, and the device was then covered with a poly(methyl methacrylate) (PMMA) resist. The cover outside of the channel region was removed such that $\mathrm{Li}$ ions in the electrolyte could intercalate from both exposed sides of the flake. During the application of the gate voltage $V_{\mathrm{G}}$, we traced the intercalation process through the measurement of the sourcedrain current (Fig. 1C). The forward scan shows a sharp increase of current corresponding to sudden intercalation, possibly owing to a shift of the layers causing a change in the stacking pattern (16). In contrast, the deintercalation in the reverse scan is rather gradual. This process enabled us to control the intercalation level down to the lowest level of $x=0.0038$. The Hall effect showed a systematic dependence on $V_{\mathrm{G}}$ (Fig. 1D) and was almost independent of temperature and measurement position on the flake (figs. S2 and S3). To determine the Li concentration $x$, we used the Hall carrier density at $150 \mathrm{~K}$ and assumed that one electron per Li-ion is supplied to the $\mathrm{ZrNCl}$ conduction layer.

In Fig. 2, A and B, we show the temperature dependence of resistivity at various doping levels. The SC $T_{\mathrm{c}}$ increased from $11.5 \mathrm{~K}$ to $19.0 \mathrm{~K}$ by reducing the doping level from 0.28 to 0.011 . We defined $T_{\mathrm{c}}$ as the midpoint of the resistive transition. The maximum $T_{\mathrm{c}}$ of $19.0 \mathrm{~K}$ is higher than the previously reported value $(15.4 \mathrm{~K})$ for the $\mathrm{ZrNCl}$ system $(15,17)$; interestingly, this is realized by reducing the doping level. Below $x=0.011$, corresponding to a carrier density of $n_{3 \mathrm{D}}=2.1 \times 10^{20} \mathrm{~cm}^{-3}, T_{\mathrm{c}}$ starts to decrease, leading to a peak structure in the phase diagram (see Fig. 3E).

The resistive transition in the highly doped regime is sharp, whereas it is substantially broadened in the lightly doped regime (Fig. 2B). As reported previously (15), this feature represents a dimensional crossover from anisotropic 3D to $2 \mathrm{D}$ SC because of the reduced interlayer hopping, as discussed later. In the $2 \mathrm{D}$ regime, the superconducting transition is described by the BKT transition, and the temperature dependence of resistivity follows the Halperin-Nelson equation $(24,25)$.

$$
\rho(T)=a \rho_{\mathrm{N}} \exp \left\{-2\left[\frac{b\left(T_{\mathrm{c}}^{\prime}-T\right)}{T-T_{\mathrm{BKT}}}\right]^{1 / 2}\right\}
$$

where $a, b$, and $T_{\mathrm{c}}{ }^{\prime}$ are fitting parameters, and $\rho_{\mathrm{N}}$ is resistivity in the normal state. According to this formula, [d(ln $\rho) /$ $\mathrm{d} T]^{-2 / 3}$ is proportional to $\left(T-T_{\mathrm{BKT}}\right)$ around $T_{\mathrm{BKT}}$, from which we determine $T_{\text {ВКт }}$ as the temperature-axis intercept of the

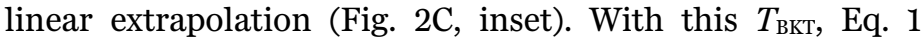
provides a good fit in the transition region for $x=0.011$ (black line in Fig. $2 \mathrm{C}$, where $T_{\mathrm{BKT}}=17.9 \mathrm{~K}, a \rho_{\mathrm{N}}=1.59 \mathrm{~m} \Omega$ $\mathrm{cm}, b=1.23$, and $\left.T_{\mathrm{c}}{ }^{\prime}=20.1 \mathrm{~K}\right)$. This resistivity fit to Eq. 1 holds at least up to $x=0.045$, but not above 0.13 due to the sharp transition. In the previous paper, the two- 
dimensionality of the SC was also confirmed at $x=0.06$ by the temperature dependence of the in-plane upper critical field, $H_{\mathrm{c} 2}^{\|}(T) \propto \sqrt{1-T / T_{\mathrm{c}}}$, which is typical behavior for $2 \mathrm{D}$ superconductors explained within the Ginzburg-Landau (GL) model [figure 2C of (15)]. These results lead us to conclude that the crossover from anisotropic $3 \mathrm{D}$ to $2 \mathrm{D} \mathrm{SC}$ occurs around $x \sim 0.1$.

The dimensional crossover from anisotropic 3D to 2D $\mathrm{SC}$ caused by the reduction of carrier density is a unique and unexpected phenomenon, particularly when we recall that the out-of-plane lattice parameter decreases with decreasing Li concentration (17). This feature should be attributed to the peculiar nature of rhombohedral stacking of $\mathrm{ZrNCl}$ layers, where the unit cell is composed of 3 layers (Fig. 1A). According to symmetry arguments, the interlayer hopping at the $\mathrm{K}$ point of the hexagonal Brillouin zone becomes exactly zero up to the second nearest layer in the rhombohedral structure (26). Our density functional theory (DFT) calculation with improved energy resolution also confirms the indiscernible $K-H$ dispersion (27). When the doping level is reduced, the Fermi surface converges to the Kpoint. Consequently, the interlayer coupling is weakened, and the out-of-plane coherence length is significantly shortened.

Figure 2D shows the temperature dependence of the out-of-plane upper critical field $H_{\mathrm{c} 2}$ at each doping level (see fig. S4 for the determination of $H_{\mathrm{c} 2}$ ). Based on the GL model, $\mu_{0} H_{\mathrm{c} 2}(T)=\Phi_{0} /\left(2 \pi \xi^{2}\right)\left(1-T / T_{\mathrm{c}}\right)$ with $\Phi_{0}$ a flux quantum, we derived the in-plane coherence length at zero-temperature ( $\xi$ ) by using the slope of the $\mu_{0} H_{\mathrm{c} 2}-T$ line (Fig. 2E). Even after $T_{\mathrm{c}}$ starts to drop below $x=0.011$, $\xi$ keeps decreasing, indicating that strongly coupled small Cooper pairs are realized in the low carrier density regime.

During the cooling process for the transport measurements, we were able to perform tunneling spectroscopy, thanks to the formation of a Schottky barrier at the electrode interface which served as the tunneling barrier (15). Figure $3 \mathrm{~A}$ displays symmetrized tunneling spectra $\mathrm{d} I / \mathrm{d} V$ at $T=2 \mathrm{~K}$ (see fig. S5 for the raw data). We normalized the spectra at $55 \mathrm{~K}$ after the subtraction of the channel resistance contribution (15). The superconducting gap structure is clearly observed and becomes wider in the low doping regime. The gap energy $\Delta$ is determined by fitting the spectra to the Dynes function (28), and its doping dependence is plotted in Fig. 3B. At high doping levels, the present results agree well with the previously reported values determined by specific heat measurements on bulk polycrystalline samples (29). The value of $2 \Delta / k_{\mathrm{B}} T_{\mathrm{c}}$ is around 3.5 at the highest doping level, indicating that SC in the highly doped state is within the BCS regime. Decreasing carrier density corresponds to stronger coupling, which is reflected in a dramatic rise of $2 \Delta / k_{\mathrm{B}} T_{\mathrm{c}}$, reaching 6.0 at the lowest carrier density.

We show the temperature evolution of the tunneling spectra at a low-doping level in Fig. 3C. The gap smoothly evolves and, importantly, closes only at temperatures higher than $T_{\text {c. }}$. The inset of Fig. 3C displays the temperature dependence of the zero-bias conductance (ZBC), $\mathrm{d} I / \mathrm{d} V$ at $V=$ 0 . We determined the gap-opening temperature $T^{*}$ by a $1 \%$ drop of the $\mathrm{ZBC}$ from the value at high temperatures. Figure 3D shows the temperature dependence of $\Delta$ at $x=0.0066$ and 0.13 (see fig. S7 for further information). We set $\Delta=0$ at temperatures above $T^{*}$, and the scaled BCS-type gap function was calculated by using $T_{\mathrm{c}}$. For $x=0.0066, T^{*}$ is more than twice as high as $T_{\mathrm{c}}$, demonstrating the stabilization of the pseudogap state. This is in marked contrast with the case of $x=0.13$, where $T_{\mathrm{c}}$ and $T^{*}$ match each other. Such behavior is summarized in the phase diagram, Fig. 3E, showing that the pseudogap state is significantly developed in the low carrier density regime.

In Fig. 4A, we show the doping dependence of $\Delta / E_{\mathrm{F}}$ and $1 /\left(k_{\mathrm{F}} \xi\right)$, where $k_{\mathrm{F}}$ is the Fermi wave vector. $\Delta / E_{\mathrm{F}}$ can be converted to the dimensionless crossover parameter $\ln \left(k_{\mathrm{F}} a_{2 \mathrm{D}}\right)$, where $a_{2 \mathrm{D}}$ is the 2D scattering length (22). The crossover regime in $2 \mathrm{D}$ systems corresponds to $\ln \left(k_{\mathrm{F}} a_{2 \mathrm{D}}\right)>2$ and $\Delta / E_{\mathrm{F}}$ $>0.3$. Larger value of $1 /\left(k_{\mathrm{F}} \xi\right)$ represents a smaller number of overlapped pairs since $1 / k_{\mathrm{F}}$ represents the interparticle distance. $E_{\mathrm{F}}$ and $k_{\mathrm{F}}$ are estimated by the carrier density and the doping-independent effective mass of $\mathrm{ZrNCl}, 0.9 m_{0}$, which was extracted from the specific heat measurements (29). This effective mass is also supported by the DFT calculation combined with electron-phonon interactions (27). The monotonic increase in $\Delta / E_{\mathrm{F}}$ and $1 /\left(k_{\mathrm{F}} \xi\right)$ toward the low doping limit represent the continuous shift from the BCS limit toward the BCS-BEC crossover regime. Importantly, $\Delta / E_{\mathrm{F}}$ becomes larger than 0.3 at $x=0.005$, corresponding to the BCS-BEC crossover region $(22) .1 /\left(k_{\mathrm{F}} \xi\right) \sim 0.36$ means that a few Cooper pairs overlap, being distinct from the assumption in the BCS theory.

By taking $\Delta / E_{\mathrm{F}}$ or $1 /\left(k_{\mathrm{F}} \xi\right)$ as a bottom axis, we depict the BCS-BEC crossover phase diagram (Fig. 4B). Both the superconducting and the pseudogap phase systematically evolve as predicted by theories $(2,7)$. Because the $2 \mathrm{D} \mathrm{SC}$ is realized in the lightly doped regime, it should be compared to the theory developed for $2 \mathrm{D}$ systems, where $T_{\mathrm{BKT}} / T_{\mathrm{F}}=0.125$ is derived as the upper bound for the transition in all fermion systems (23). The present measurements reached the value of $T_{\mathrm{BKT}} / T_{\mathrm{F}}=0.116\left(T_{\mathrm{c}} / T_{\mathrm{F}}=0.121\right)$ at $\Delta / E_{\mathrm{F}}=0.36$, which is close to the theoretical limit. This agreement demonstrates that superconductors, where charged electron pairs reside in lattice potentials, can reach the general upper bound. Figure $4 \mathrm{C}$ shows the Uemura plot of our results together 
with other materials $(11,13,15,30-32) . \mathrm{Li}_{x} \mathrm{ZrNCl}$ approaches the limit of $T_{\mathrm{c}} / T_{\mathrm{F}}=0.125$ with decreasing $T_{\mathrm{F}}$ from the BCS limit (lower right side of the plot), providing a strong piece of evidence that the BCS-BEC crossover is indeed realized. The $T_{\mathrm{c}}$ reduction below $x=0.011$ (Fig. $3 \mathrm{E}$ ) should be attributed to the limitation of $T_{\mathrm{c}}\left(T_{\mathrm{BKT}}\right)$ scaled by $T_{\mathrm{F}}$ in the BCS-BEC crossover regime rather than to other causes, such as a tendency toward electron localization.

Here, it is fruitful to discuss pseudogap states in several materials and compare them with the present system. In unconventional superconductors, pseudogap states are frequently observed close to insulating phases, but they tend to be complex because of strong electron correlation effects and magnetic ordering in many cases (9). In stark contrast, $\mathrm{Li}_{x} \mathrm{ZrNCl}$ offers a much simpler testbed because the undoped state at $x=0$ is a band-insulator free from electron correlation effects, magnetic orders, and density waves. Also, unlike magic-angle twisted bilayer graphene, the simple parabolic conduction band does not apply further constraints on $T_{\mathrm{c}}(23)$ and makes $\mathrm{Li}_{x} \mathrm{ZrNCl}$ a model system for the BCS-BEC crossover.

Even in disordered thin films, the pseudogap state is observed owing to localized Cooper pairs (33). Such a localized model is unlikely for the present system because resistivity shows metallic behavior. Furthermore, as summarized in table $\mathrm{S} 1$, the mean free path in the $\mathrm{Li}_{x} \mathrm{ZrNCl}$ system is comparable to the Pippard coherence length $\xi_{\text {pippard }}=\hbar v_{\mathrm{F}} /$ $\pi \Delta$, where $\hbar$ and $v_{\mathrm{F}}$ are Dirac's constant and the Fermi velocity, respectively, indicating that the system is far from the dirty limit.

These considerations strongly support the notion that the observed pseudogap state in $\mathrm{Li}_{x} \mathrm{ZrNCl}$ can be attributed to the preformed pair formation in the BCS-BEC crossover scenario. We note that the pseudogap state is observable in a wide range, even when $\Delta / E_{\mathrm{F}}$ is less than 0.3. In a bulk study, an NMR measurement on polycrystalline $\mathrm{Li}_{x} \mathrm{ZrNCl}$ samples reported a pseudogap state on the high doping side of the superconducting dome (34). $T^{*}$ was reported to be $\sim 25 \mathrm{~K}$ when $T_{\mathrm{c}}=15 \mathrm{~K}(x=0.08)$, which agrees with the present result. The early appearance of pseudogap states below $x \sim 0.1$ may be attributed to two-dimensionality, which prefers the pseudogap formation (35). This scenario can be applied to the pseudogap state observed at a $\mathrm{LaAlO}_{3} / \mathrm{SrTiO}_{3}$ interface (36). That system is distant from the BCS-BEC crossover regime (Fig. 4C), but its two-dimensionality gives rise to pseudogap.

We have demonstrated a 2D BCS-BEC crossover through the systematic tuning of the coupling strength of SC in $\mathrm{Li}_{x} \mathrm{ZrNCl}$. The 2D BCS-BEC crossover was realized in the present system owing to the dimensional crossover from anisotropic $3 \mathrm{D}$ to $2 \mathrm{D}$ upon reducing the carrier density. This crossover should be compared to arrays of $2 \mathrm{D}$ clouds of
Fermi gases (37) where dimensionality is also affected by the coupling strength. Such complementary studies advance our understanding of fermion condensation physics.

\section{REFERENCES AND NOTES}

1. D. M. Eagles, Possible pairing without superconductivity at low carrier concentrations in bulk and thin-film superconducting semiconductors. Phys. Rev. 186, 456-463 (1969). doi:10.1103/PhysRev.186.456

2. C. A. R. Sá de Melo, M. Randeria, J. R. Engelbrecht, Crossover from BCS to Bose superconductivity: Transition temperature and time-dependent GinzburgLandau theory. Phys. Rev. Lett. 71, 3202-3205 (1993). doi:10.1103/PhysRevLett.71.3202 Medline

3. M. Greiner, C. A. Regal, D. S. Jin, Emergence of a molecular Bose-Einstein condensate from a Fermi gas. Nature 426, 537-540 (2003). doi:10.1038/nature02199 Medline

4. C. A. Regal, M. Greiner, D. S. Jin, Observation of resonance condensation of fermionic atom pairs. Phys. Rev. Lett. 92, 040403 (2004). doi:10.1103/PhysRevLett.92.040403 Medline

5. A. Gezerlis, J. Carlson, Low-density neutron matter. Phys. Rev. C 81, 025803 (2010). doi:10.1103/PhysRevC.81.025803

6. J. P. Gaebler, J. T. Stewart, T. E. Drake, D. S. Jin, A. Perali, P. Pieri, G. C. Strinati, Observation of pseudogap behaviour in a strongly interacting Fermi gas. Nat. Phys. 6, 569-573 (2010). doi:10.1038/nphys1709

7. M. Randeria, E. Taylor, Crossover from Bardeen-Cooper-Schrieffer to BoseEinstein condensation and the unitary Fermi gas. Annu. Rev. Condens. Matter Phys. 5, 209-232 (2014). doi:10.1146/annurev-conmatphys-031113-133829

8. Y. J. Uemura, L. P. Le, G. M. Luke, B. J. Sternlieb, W. D. Wu, J. H. Brewer, T. M. Riseman, C. L. Seaman, M. B. Maple, M. Ishikawa, D. G. Hinks, J. D. Jorgensen, G. Saito, H. Yamochi, Basic similarities among cuprate, bismuthate, organic, Chevrel-phase, and heavy-fermion superconductors shown by penetrationdepth measurements. Phys. Rev. Lett. 66, 2665-2668 (1991). doi:10.1103/PhysRevLett.66.2665 Medline

9. B. Keimer, S. A. Kivelson, M. R. Norman, S. Uchida, J. Zaanen, From quantum matter to high-temperature superconductivity in copper oxides. Nature 518 , 179-186 (2015). doi:10.1038/nature14165 Medline

10. S. Rinott, K. B. Chashka, A. Ribak, E. D. L. Rienks, A. Taleb-Ibrahimi, P. Le Fevre, F. Bertran, M. Randeria, A. Kanigel, Tuning across the BCS-BEC crossover in the multiband superconductor $\mathrm{Fe}_{1+y} \mathrm{Se}_{x} \mathrm{Te}_{1-x}$ : An angle-resolved photoemission study. Sci. Adv. 3, e1602372 (2017). doi:10.1126/sciadv.1602372 Medline

11. S. Kasahara, T. Watashige, T. Hanaguri, Y. Kohsaka, T. Yamashita, Y. Shimoyama, Y. Mizukami, R. Endo, H. Ikeda, K. Aoyama, T. Terashima, S. Uji, T. Wolf, H. von Löhneysen, T. Shibauchi, Y. Matsuda, Field-induced superconducting phase of FeSe in the BCS-BEC cross-over. Proc. Natl. Acad. Sci. U.S.A. 111, 16309-16313 (2014). doi:10.1073/pnas.1413477111 Medline

12. T. Hashimoto, Y. Ota, A. Tsuzuki, T. Nagashima, A. Fukushima, S. Kasahara, Y Matsuda, K. Matsuura, Y. Mizukami, T. Shibauchi, S. Shin, K. Okazaki, BoseEinstein condensation superconductivity induced by disappearance of the nematic state. Sci. Adv. 6, eabb9052 (2020). doi:10.1126/sciadv.abb9052 Medline

13. Y. Cao, V. Fatemi, S. Fang, K. Watanabe, T. Taniguchi, E. Kaxiras, P. JarilloHerrero, Unconventional superconductivity in magic-angle graphene superlattices. Nature 556, 43-50 (2018). doi:10.1038/nature26160 Medline

14. J. M. Park, Y. Cao, K. Watanabe, T. Taniguchi, P. Jarillo-Herrero, Tunable strongly coupled superconductivity in magic-angle twisted trilayer graphene. Nature 590 , 249-255 (2021). doi:10.1038/s41586-021-03192-0

15. Y. Nakagawa, Y. Saito, T. Nojima, K. Inumaru, S. Yamanaka, Y. Kasahara, Y. Iwasa, Gate-controlled low carrier density superconductors: Toward the twodimensional BCS-BEC crossover. Phys. Rev. B 98, 064512 (2018). doi:10.1103/PhysRevB.98.064512 
16. Y. Kasahara, K. Kuroki, S. Yamanaka, Y. Taguchi, Unconventional superconductivity in electron-doped layered metal nitride halides $M N X(M=T$, $\mathrm{Zr}, \mathrm{Hf} ; X=\mathrm{Cl}, \mathrm{Br}, \mathrm{I})$. Physica C 514, 354-367 (2015). doi:10.1016/i.physc.2015.02.022

17. Y. Taguchi, A. Kitora, Y. Iwasa, Increase in $T_{\mathrm{c}}$ upon reduction of doping in $\mathrm{Li}_{\mathrm{x}} \mathrm{ZrNCl}$ superconductors. Phys. Rev. Lett. 97, 107001 (2006) doi:10.1103/PhysRevLett.97.107001 Medline

18. K. Kuroki, Spin-fluctuation-mediated $d+i d^{\prime}$ pairing mechanism in doped $\beta-M N C l$ ( $M=\mathrm{Hf}, \mathrm{Zr}$ ) superconductors. Phys. Rev. B 81, 104502 (2010). doi:10.1103/PhysRevB.81.104502

19. M. Calandra, P. Zoccante, F. Mauri, Universal increase in the superconducting critical temperature of two-dimensional semiconductors at low doping by the electron-electron interaction. Phys. Rev. Lett. 114, 077001 (2015). doi:10.1103/PhysRevLett.114.077001 Medline

20. J. T. Ye, Y. J. Zhang, R. Akashi, M. S. Bahramy, R. Arita, Y. Iwasa, Superconducting dome in a gate-tuned band insulator. Science 338, 1193-1196 (2012). doi:10.1126/science.1228006 Medline

21. Y. Saito, Y. Kasahara, J. Ye, Y. Iwasa, T. Nojima, Metallic ground state in an iongated two-dimensional superconductor. Science 350, 409-413 (2015). doi:10.1126/science.1259440 Medline

22. L. He, H. Lü, G. Cao, H. Hu, X.-J. Liu, Quantum fluctuations in the BCS-BEC crossover of two-dimensional Fermi gases. Phys. Rev. A 92, 023620 (2015) doi:10.1103/PhysRevA.92.023620

23. T. Hazra, N. Verma, M. Randeria, Bounds on the superconducting transition temperature: Applications to twisted bilayer graphene and cold atoms. Phys. Rev. X 9, 031049 (2019). doi:10.1103/PhysRevX.9.031049

24. B. I. Halperin, D. R. Nelson, Resistive transition in superconducting films. J. Low Temp. Phys. 36, 599-616 (1979). doi:10.1007/BF00116988

25. A. M. Kadin, K. Epstein, A. M. Goldman, Renormalization and the KosterlitzThouless transition in a two-dimensional superconductor. Phys. Rev. B 27, 66916702 (1983). doi:10.1103/PhysRevB.27.6691

26. R. Akashi, Y. lida, K. Yamamoto, K. Yoshizawa, Interference of the Bloch phase in layered materials with stacking shifts. Phys. Rev. B 95, 245401 (2017). doi:10.1103/PhysRevB.95.245401

27. See supplementary materials.

28. R. C. Dynes, V. Narayanamurti, J. P. Garno, Direct measurement of quasiparticlelifetime broadening in a strong-coupled superconductor. Phys. Rev. Lett. 41, 1509-1512 (1978). doi:10.1103/PhysRevLett.41.1509

29. Y. Kasahara, T. Kishiume, T. Takano, K. Kobayashi, E. Matsuoka, H. Onodera, K. Kuroki, Y. Taguchi, Y. Iwasa, Enhancement of pairing interaction and magnetic fluctuations toward a band insulator in an electron-doped $\mathrm{Li} \times \mathrm{ZrNCl}$ Superconductor. Phys. Rev. Lett. 103, 077004 (2009). doi:10.1103/PhysRevLett.103.077004 Medline

30. Q.-W. Wang, Z. Li, W.-H. Zhang, Z.-C. Zhang, J.-S. Zhang, W. Li, H. Ding, Y.-B. Ou, P. Deng, K. Chang, J. Wen, C.-L. Song, K. He, J.-F. Jia, S.-H. Ji, Y.-Y. Wang, L.-L. Wang, X. Chen, X.-C. Ma, Q.-K. Xue, Interface-Induced High-Temperature Superconductivity in Single Unit-Cell FeSe Films on $\mathrm{SrTiO}_{3}$. Chin. Phys. Lett. 29 037402 (2012). doi:10.1088/0256-307X/29/3/037402

31. Y. J. Uemura, Condensation, excitation, pairing, and superfluid density in high- $T_{\mathrm{c}}$ superconductors: The magnetic resonance mode as a roton analogue and a possible spin-mediated pairing. J. Phys. Condens. Matter 16, S4515-S4540 (2004). doi:10.1088/0953-8984/16/40/007

32. X. Lin, Z. Zhu, B. Fauqué, K. Behnia, Fermi surface of the most dilute superconductor. Phys. Rev. X 3, 021002 (2013). doi:10.1103/PhysRevX.3.021002

33. B. Sacépé, T. Dubouchet, C. Chapelier, M. Sanquer, M. Ovadia, D. Shahar, M. Feigel'man, L. loffe, Localization of preformed Cooper pairs in disordered superconductors. Nat. Phys. 7, 239-244 (2011). doi:10.1038/nphys1892

34. H. Kotegawa, S. Oshiro, Y. Shimizu, H. Tou, Y. Kasahara, T. Kishiume, Y. Taguchi, Y. Iwasa, Strong suppression of coherence effect and appearance of pseudogap in the layered nitride superconductor $\mathrm{Li}_{x} \mathrm{ZrNCl}:{ }^{91} \mathrm{Zr}$ - and ${ }^{15} \mathrm{~N}-\mathrm{NMR}$ studies. Phys. Rev. B 90, 020503(R) (2014). doi:10.1103/PhysRevB.90.020503

35. Q. Chen, I. Kosztin, B. Jankó, K. Levin, Superconducting transitions from the pseudogap state: $D$-wave symmetry, lattice, and low-dimensional effects. Phys. Rev. B 59, 7083-7093 (1999). doi:10.1103/PhysRevB.59.7083

36. C. Richter, H. Boschker, W. Dietsche, E. Fillis-Tsirakis, R. Jany, F. Loder, L. F. Kourkoutis, D. A. Muller, J. R. Kirtley, C. W. Schneider, J. Mannhart, Interface superconductor with gap behaviour like a high-temperature superconductor Nature 502, 528-531 (2013). doi:10.1038/nature12494 Medline

37. M. G. Ries, A. N. Wenz, G. Zürn, L. Bayha, I. Boettcher, D. Kedar, P. A. Murthy, M. Neidig, T. Lompe, S. Jochim, Observation of pair condensation in the quasi-2D BEC-BCS crossover. Phys. Rev. Lett. 114, 230401 (2015). doi:10.1103/PhysRevLett.114.230401 Medline

38. Harvard Dataverse, https://doi.org/10.7910/DVN/MJAMIV.

39. S. Yamanaka, H. Kawaji, K.-i. Hotehama, M. Ohashi, A new layer-structured nitride superconductor. Lithium-intercalated $\beta$-zirconium nitride chloride, LixZrNCl. Adv. Mater. 8, 771-774 (1996). doi:10.1002/adma.19960080917

40. J. K. Hulm, C. K. Jones, D. W. Deis, H. A. Fairbank, P. A. Lawless, Superconducting Interactions in Tin Telluride. Phys. Rev. 169, 388-394 (1968) doi:10.1103/PhysRev.169.388

41. T. D. Thanh, A. Koma, $\mathrm{S}$. Tanaka, Superconductivity in the $\mathrm{BaPb}_{1-x} \mathrm{Bi}_{x} \mathrm{O}_{3}$ system. Appl. Phys. 22, 205-212 (1980). doi:10.1007/BF00886008

42. T. Takano, A. Kitora, Y. Taguchi, Y. Iwasa, Modulation-doped-semiconductorlike behavior manifested in magnetotransport measurements of $\mathrm{Li} \mathrm{i}_{\mathrm{Z}} \mathrm{rNCl}$ layered superconductors. Phys. Rev. B 77, 104518 (2008) doi:10.1103/PhysRevB.77.104518

43. R. Akashi, M. Ochi, R. Suzuki, S. Bordács, Y. Tokura, Y. Iwasa, R. Arita, TwoDimensional Valley Electrons and Excitons in Noncentrosymmetric $3 R-\mathrm{MoS}_{2}$. Phys. Rev. Appl. 4, 014002 (2015). doi:10.1103/PhysRevApplied.4.014002

44. R. Weht, A. Filippetti, W. E. Pickett, Electron doping in the honeycomb bilayer superconductors ( $\mathrm{Zr}$, Hf) NCl. Europhys. Lett. 48, 320-325 (1999). doi:10.1209/epl/i1999-00484-4

45. R. Heid, K. P. Bohnen, Ab Initio lattice dynamics and electron-phonon coupling in Li $i_{x}$ rNCl. Phys. Rev. B 72, 134527 (2005). doi:10.1103/PhysRevB.72.134527

46. M. Lüders, M. A. L. Marques, N. N. Lathiotakis, A. Floris, G. Profeta, L. Fast, A. Continenza, S. Massidda, E. K. U. Gross, Ab initio theory of superconductivity. I. Density functional formalism and approximate functionals. Phys. Rev. B 72. 024545 (2005). doi:10.1103/PhysRevB.72.024545

47. M. Kawamura, R. Akashi, S. Tsuneyuki, Anisotropic superconducting gaps in $\mathrm{YNi}_{2} \mathrm{~B}_{2} \mathrm{C}$ : A first-principles investigation. Phys. Rev. B 95, 054506 (2017). doi:10.1103/PhysRevB.95.054506

48. R. Akashi, K. Nakamura, R. Arita, M. Imada, High-temperature superconductivity in layered nitrides $\beta-\mathrm{Lix} M N C l(M=T i, Z r, H f)$ : Insights from density functional theory for superconductors. Phys. Rev. B 86, 054513 (2012). doi:10.1103/PhysRevB.86.054513

49. P. Giannozzi, S. Baroni, N. Bonini, M. Calandra, R. Car, C. Cavazzoni, D. Ceresoli, G. L. Chiarotti, M. Cococcioni, I. Dabo, A. Dal Corso, S. de Gironcoli, S. Fabris, G. Fratesi, R. Gebauer, U. Gerstmann, C. Gougoussis, A. Kokalj, M. Lazzeri, L. Martin-Samos, N. Marzari, F. Mauri, R. Mazzarello, S. Paolini, A. Pasquarello, L. Paulatto, C. Sbraccia, S. Scandolo, G. Sclauzero, A. P. Seitsonen, A. Smogunov, P. Umari, R. M. Wentzcovitch, QUANTUM ESPRESSO: A modular and opensource software project for quantum simulations of materials. J. Phys. Condens. Matter 21, 395502 (2009). doi:10.1088/0953-8984/21/39/395502 Medline

50. P. Giannozzi, O. Andreussi, T. Brumme, O. Bunau, M. Buongiorno Nardelli, M. Calandra, R. Car, C. Cavazzoni, D. Ceresoli, M. Cococcioni, N. Colonna, I. Carnimeo, A. Dal Corso, S. de Gironcoli, P. Delugas, R. A. DiStasio Jr., A. Ferretti, A. Floris, G. Fratesi, G. Fugallo, R. Gebauer, U. Gerstmann, F. Giustino, T. Gorni, J. Jia, M. Kawamura, H.-Y. Ko, A. Kokalj, E. Küçükbenli, M. Lazzeri, M. Marsili, N. Marzari, F. Mauri, N. L. Nguyen, H.-V. Nguyen, A. Otero-de-la-Roza, L. Paulatto, S. Poncé, D. Rocca, R. Sabatini, B. Santra, M. Schlipf, A. P. Seitsonen, A. 
Smogunov, I. Timrov, T. Thonhauser, P. Umari, N. Vast, X. Wu, S. Baroni,

Advanced capabilities for materials modelling with Quantum ESPRESSO. J. Phys.

Condens. Matter 29, 465901 (2017). doi:10.1088/1361-648X/aa8f79 Medline

51. D. R. Hamann, Optimized norm-conserving Vanderbilt pseudopotentials. Phys. Rev. B 88, 085117 (2013). doi:10.1103/PhysRevB.88.085117

52. P. Scherpelz, M. Govoni, I. Hamada, G. Galli, Implementation and validation of fully relativistic GW calculations: Spin-orbit coupling in molecules, nanocrystals, and solids. J. Chem. Theory Comput. 12, 3523-3544 (2016). doi:10.1021/acs.jctc.6b00114 Medline

53. J. P. Perdew, K. Burke, M. Ernzerhof, Generalized gradient approximation made simple. Phys. Rev. Lett. 77, 3865-3868 (1996). doi:10.1103/PhysRevLett.77.3865 Medline

54. S. Baroni, S. de Gironcoli, A. Dal Corso, P. Giannozzi, Phonons and related crystal properties from density-functional perturbation theory. Rev. Mod. Phys. 73, 515562 (2001). doi:10.1103/RevModPhys.73.515

\section{ACKNOWLEDGMENTS}

We thank K. Kanoda, T. Shibauchi, T. Hanaguri, M. Nakano, R. Akashi, M. Hirayama, and F. Qin for fruitful discussions. We also thank M. Heyl for critical reading and correction of the manuscript. Funding: This work was supported by A3 Foresight Program and JSPS KAKENHI Grant Numbers JP19H05602 and JP17J08941. Y.N. was supported by the Materials Education program for the future leaders in Research, Industry, and Technology (MERIT). Author contributions: Y. N., T. N. and Y. I. conceived and designed the experiments. Y.N. fabricated the devices, performed measurements, and analyzed the data. Y.K. grew the single crystal of ZrNCl. T. N. and R. A. conducted the density functional theory calculations. All authors discussed the results and wrote the manuscript. Competing interests: Authors declare no competing interests. Data and materials availability: Data shown in the main text and the supplementary materials and instructions for the calculations are available at (38).

\section{SUPPLEMENTARY MATERIALS}

science. sciencemag.org/cgi/content/full/science.abb9860/DC1

Materials and Methods

Supplementary Text

Figs. S1 to S8

Table S1

References (39-54)

31 March 2020; accepted 5 March 2021

Published online 18 March 2021

10.1126/science.abb9860 
A

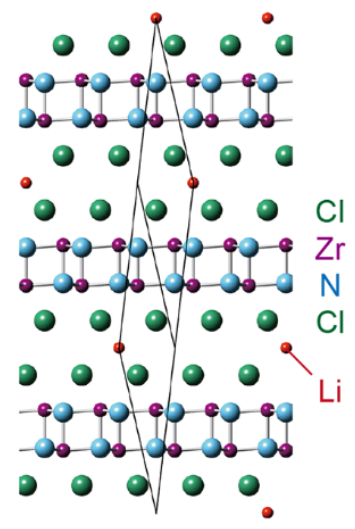

B

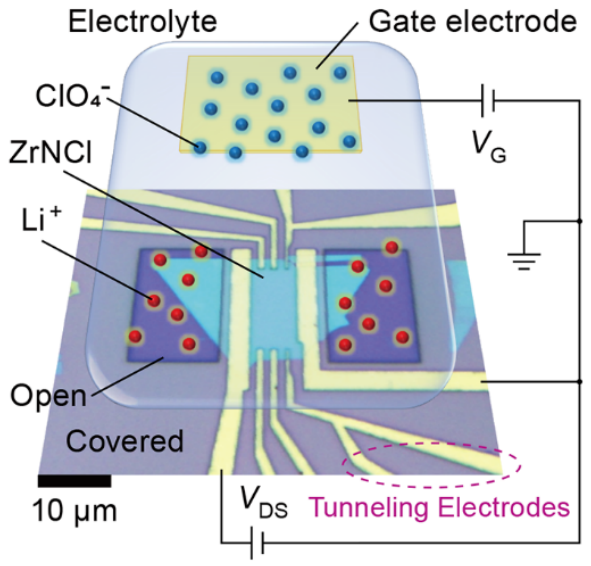

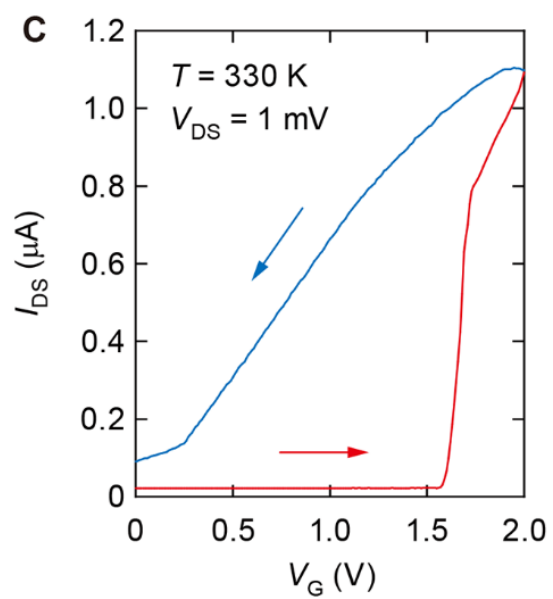

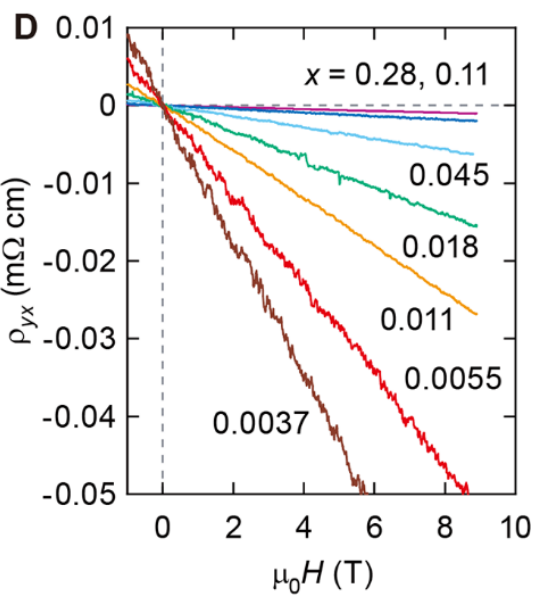

Fig. 1. Gate controlled intercalation in $\mathrm{ZrNCl}$ device. (A) Side view of $\mathrm{Li}_{x} \mathrm{ZrNCl}$ crystal structure. Solid lines represent the rhombohedral unit cell. (B) Schematic illustration of the ionic-gating device based on a real optical micrograph picture of a $\mathrm{ZrNCl}$ single crystal flake and patterned electrodes. Narrow contacts are prepared for the tunneling spectroscopy measurements. PMMA covers the whole device except for the outer area of the flake and the gate electrode. The electrolyte containing $\mathrm{LiClO}_{4}$ is dropped on the device. Gate voltage $V_{G}$ is applied to the electrolyte, and lithium cations and $\mathrm{ClO}_{4}$ anions move oppositely. Lithium cations intercalate from the sides of the flake. (C) Source-drain current $I_{D S}$ of the device in intercalation operation. During the forward sweep of $V_{G}\left(\right.$ red), I $I_{D S}$ increases steeply, whereas the change of $I_{D S}$ is gradual in the backward scan (blue). $V_{G}$ is swept at a speed of $10 \mathrm{mV} / \mathrm{sec}$. (D) Antisymmetrized transverse resistivity at $150 \mathrm{~K}$ for various values of the Li content $x$. The linear slope is used to determine $x$. 

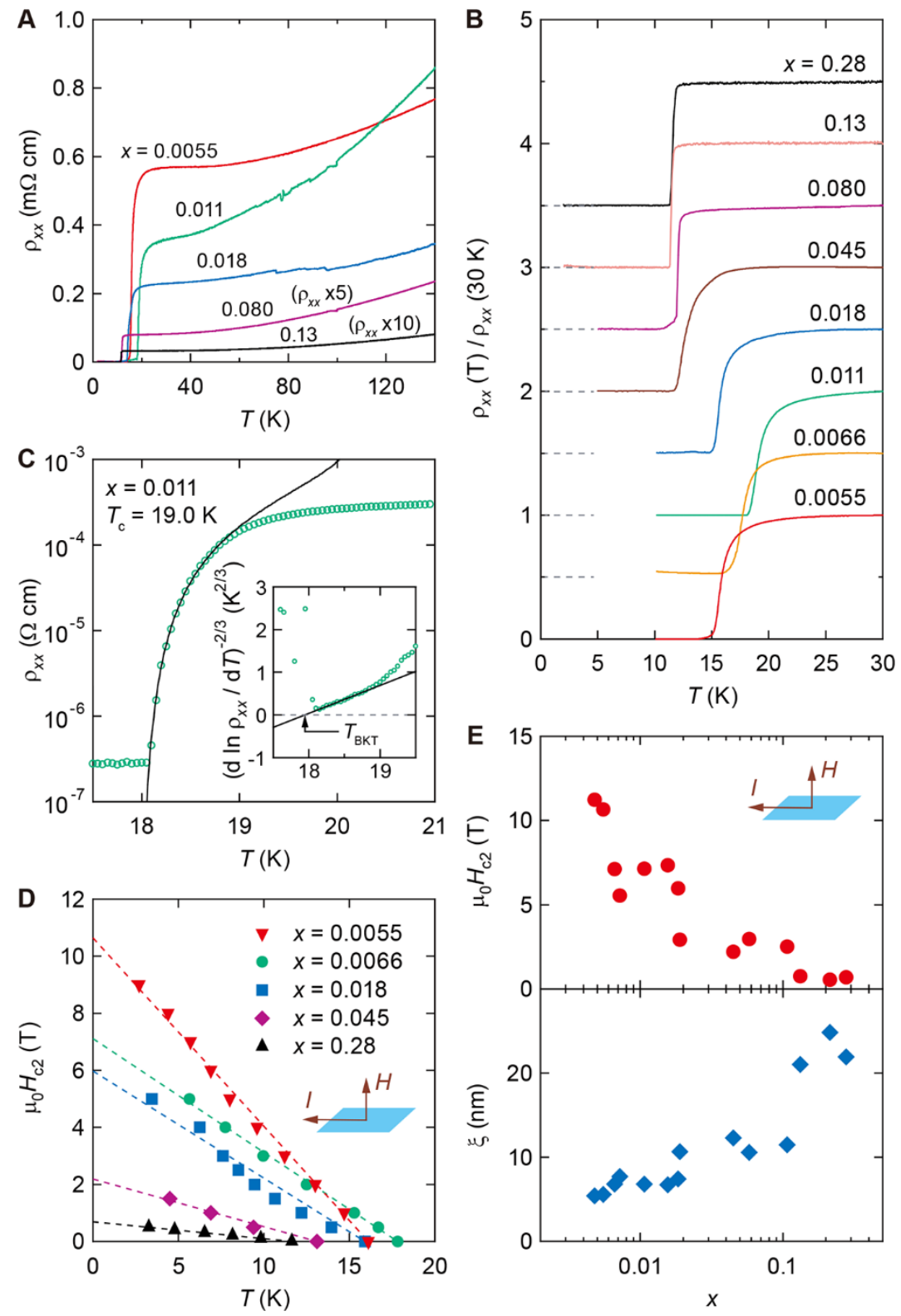

Fig. 2. Transport properties of $\mathrm{Li}_{x} \mathrm{ZrNCl}$. (A) Temperature dependence of resistivity at different doping levels. The resistivities at $x=0.080$ and 0.13 are multiplied by 5 and 10, respectively. (B) Resistivity normalized at $30 \mathrm{~K}$. Each curve is shifted by 0.5 , and gray dashed lines indicate zero lines. (C) Resistivity at $x=0.011$ showing the BKT transition. The black line is the fit to the Halperin-Nelson formula. Inset: resistivity plotted on a $[\mathrm{d}(\ln \rho) / \mathrm{d} T]^{-2 / 3}$ scale. (D) Out-of-plane upper critical field $H_{\mathrm{c} 2}$ as a function of temperature. Dashed lines are linear extrapolations to $0 \mathrm{~K}$ for each doping levels. (E) Doping dependence of $H_{\mathrm{c} 2}$ at $\mathrm{OK}$ in (D) (top) and in-plane coherence length $\xi$ (bottom). 

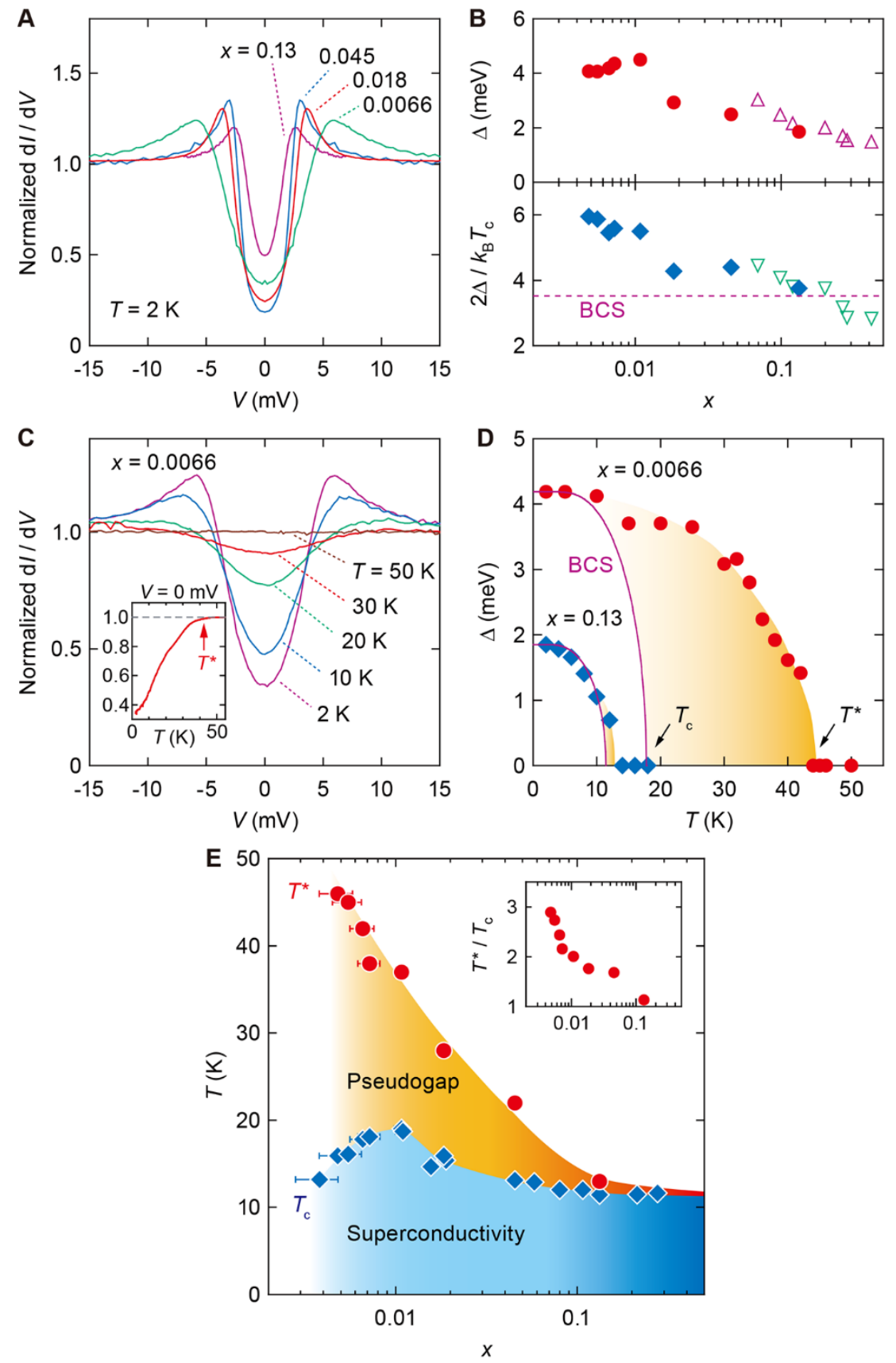

Fig. 3. Tunneling spectroscopy of $\mathrm{Li}_{x} \mathrm{ZrNCl}$. (A) Symmetrized and normalized tunneling spectra at $2 \mathrm{~K}$. At each doping level, spectra at $55 \mathrm{~K}$ are used for the normalization to remove the biasand $x$-dependent background after the subtraction of channel resistivity $(15,27)$. (B) Doping dependence of superconducting gap $\Delta$ (top) and its ratio to the critical temperature $T_{\mathrm{c}}$ (bottom). The BCS theory predicts $2 \Delta / k_{\mathrm{B}} T_{\mathrm{C}}=3.52$ (dashed line). Open symbols are measured values in polycrystalline samples (29). (C) Tunneling spectra at $x=0.0066$ for different temperatures normalized at $55 \mathrm{~K}$ without symmetrization. Inset: temperature scan of zero-bias-conductance (ZBC), $\mathrm{d} l / \mathrm{d} V$ at $V=0$. Gap-opening temperature $T^{*}$ is determined by a $1 \%$ drop of ZBC. (D) $\Delta$ at $x$ $=0.0066$ (circles) and 0.13 (diamonds) as a function of temperature. Solid lines indicate the BCStype gap function with $T_{c}$ determined by the resistive transition. (E) Phase diagram of $\mathrm{Li}_{x} \mathrm{ZrNCl}$. The temperature regime between $T_{\mathrm{c}}$ and $T^{*}$ represents the pseudogap state. The error of carrier density is estimated by measurements in multiple Hall probes. Inset: the ratio between $T^{*}$ and $T_{\mathrm{c}}$. 

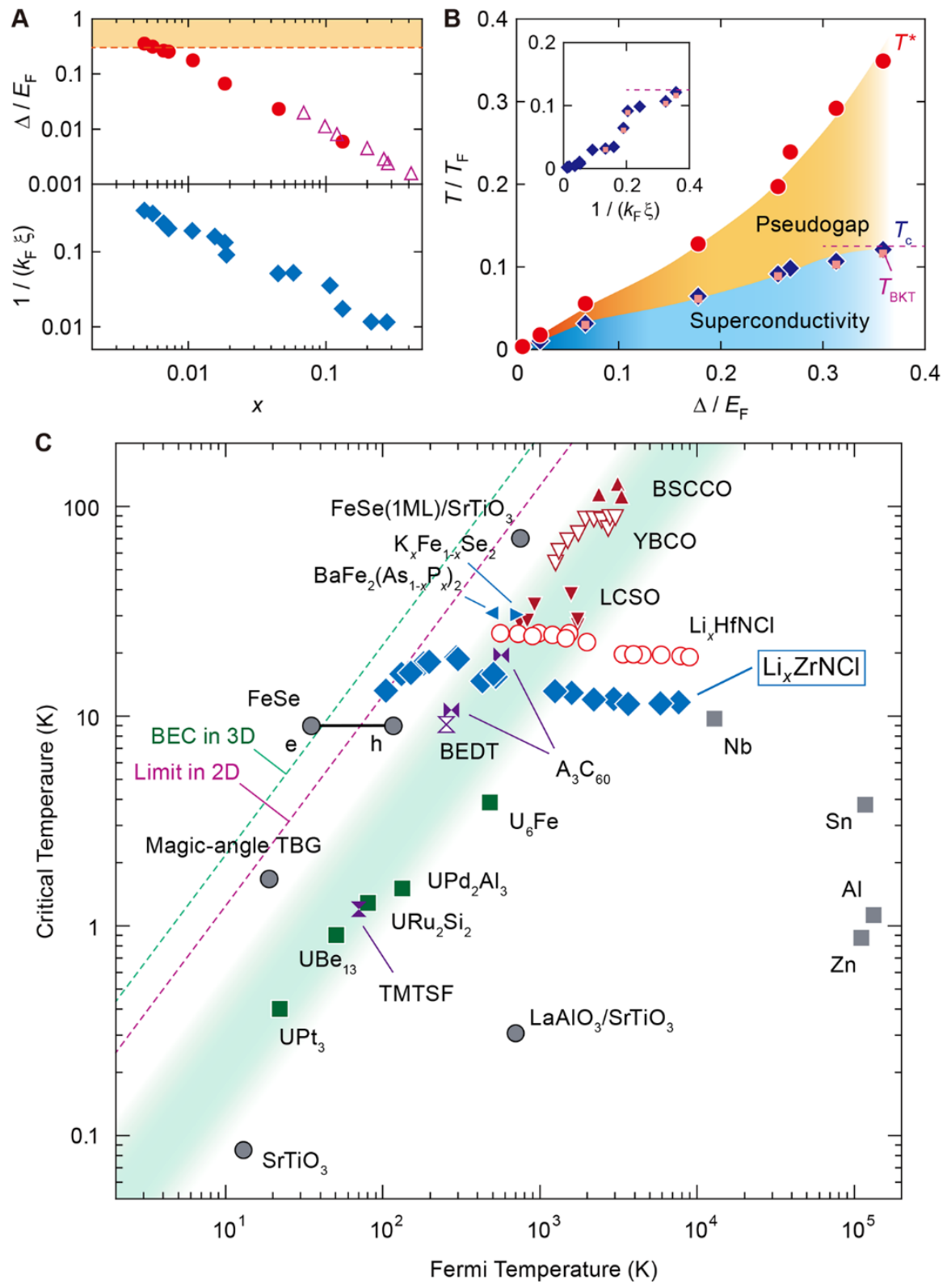

Fig. 4. The BCS-BEC crossover in superconducting $\mathrm{Li}_{x} \mathrm{ZrNCl}$. (A) Doping dependence of the ratio between superconducting gap and Fermi energy $\left(\Delta / E_{F}\right)$ (top) and the ratio between interparticle distance and coherence length $\left(1 / k_{F} \xi\right)$ (bottom). The orange area represents the BCS-BEC crossover regime (22). Open triangles are measured values from specific heat measurement (29). (B) The phase diagram of the BCS-BEC crossover. Gap opening temperature $T^{*}$, critical temperature $T_{\mathrm{c}}$ and critical temperature of BKT transition $T_{\mathrm{BKT}}$ are normalized by Fermi temperature $T_{\mathrm{F}}$ and plotted as functions of $\Delta / E_{\mathrm{F}}$ with red spheres, dark blue diamonds, and pink squares, respectively. The dashed line represents the theoretically predicted upper bound, $T_{\mathrm{BKT}} / T_{\mathrm{F}}$ $=0.125$ (23). Inset: $T_{\mathrm{C}} / T_{\mathrm{F}}$ and $T_{\mathrm{BKT}} / T_{\mathrm{F}}$ as functions of $1 / k_{\mathrm{F}} \xi$. (C) Uemura plot: Critical temperature versus Fermi temperature is plotted for various superconductors $(11,13,15,30-32)$. As $x$ is decreased, $\mathrm{Li}_{x} \mathrm{ZrNCl}$ departs from the BCS limit, arriving at the crossover region having traversed the shaded area, where most of the unconventional superconductors are located (8). The dashed line denoted as "BEC in 3D" represents the critical temperature in the BEC limit in 3D Fermi gas systems, $T_{\mathrm{c}}=0.218 T_{\mathrm{F}}(2)$. The other dashed line, denoted as "Limit in 2D", corresponds to the general upper limit of $T_{\mathrm{BKT}}=0.125 T_{\mathrm{F}}$ in all 2D fermionic systems (23). 